\title{
Targeting dePARylation for cancer therapy
}

\author{
Muzaffer Ahmad Kassab', Lily L. Yu ${ }^{2}$ and Xiaochun Yu ${ }^{1 *}$ (])
}

\begin{abstract}
Poly(ADP-ribosyl)ation (PARylation) mediated by poly ADP-ribose polymerases (PARPs) plays a key role in DNA damage repair. Suppression of PARylation by PARP inhibitors impairs DNA damage repair and induces apoptosis of tumor cells with repair defects. Thus, PARP inhibitors have been approved by the US FDA for various types of cancer treatment. However, recent studies suggest that dePARylation also plays a key role in DNA damage repair. Instead of antagonizing PARylation, dePARylation acts as a downstream step of PARylation in DNA damage repair. Moreover, several types of dePARylation inhibitors have been developed and examined in the preclinical studies for cancer treatment. In this review, we will discuss the recent progress on the role of dePARylation in DNA damage repair and cancer suppression. We expect that targeting dePARylation could be a promising approach for cancer chemotherapy in the future.
\end{abstract}

Keywords: PARG, ADP-ribosylation, dePARylation, DNA damage response, Cancer therapy

\section{Overview}

DePARylation is the process that removes ADP-ribose (ADPR) signals from various proteins during cellular stresses conditions such as DNA damage response (DDR) [1]. During DDR, ADPR moieties are attached to the substrate proteins by various poly(ADP-ribose) polymerases (PARPs) with PARP1 and PARP2 catalyzing the predominant function [2-4]. The ADP-ribosylation can just be a single ADP-ribose (mono-ADPR/MAR/MARylation) or a long chain of repetitive ADPR units (poly-ADPR/PAR/ PARylation) $[5,6]$. The PARylation signals anchor additional proteins containing PAR-binding motifs (PBMs) to the sites of damaged DNA. Thus ADP-ribosylation functions as an important post-translational modification trafficking proteins to the site of damaged DNA for DNA repair thereby helping in maintaining genomic stability $[1,3,7,8]$.

DNA damage activates PARP1/2 that in turn generates covalently attached MAR/PAR chains onto themselves (auto-PARylation) and other acceptor proteins (trans-PARylation) utilizing $\mathrm{NAD}^{+}$as an ADP-ribose

\footnotetext{
*Correspondence: xyu@coh.org

1 Department of Cancer Genetics \& Epigenetics, Beckman Research Institute, City of Hope, Duarte, CA 91010, USA

Full list of author information is available at the end of the article
}

donor and generating nicotinamide as a byproduct. PARylation modulates the function and structure of the modified proteins. The modified proteins, in turn, recruit additional proteins involved in DDR to the damaged loci $[2,9]$. PARylation is a reversible modification, and consequently, this modification is terminated and cellular homeostasis is attained. The removal of PAR chains is mainly attained due to the hydrolysis of these polymers by poly(ADP-ribose) glycohydrolase (PARG) [10, 11]. However, PARG cannot remove the terminal ADPribose and thus the complete removal of the PARylation signals requires additional enzymes [12]. The additional hydrolases include TARG1 terminal ADP-ribose protein glycohydrolase (TARG1), macrodomain containing proteins MacroD1/D2 and recently discovered ADP-riboseacceptor hydrolases ARH1/3 [1, 13-15].

Therapeutic perturbation of the PARylation/dePARylation processes has successfully demonstrated the selective killing of cancerous cells. Most notably, PARP1/ PARP2 inhibitors (PARPi) are actively used in the clinical treatments of familial breast and ovarian cancers with partial DDR defects $[16,17]$. PARPi suppresses PARP1/ PARP2 function, which in turn prevents an optimal DDR [18-20] thereby inducing cell death. However, unfortunately, like other chemo-drugs, cancers resistance to PARPi has emerged [21-23]. Recent countermeasures

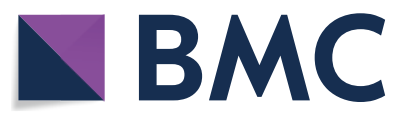

(c) The Author(s) 2020. This article is licensed under a Creative Commons Attribution 4.0 International License, which permits use, sharing, adaptation, distribution and reproduction in any medium or format, as long as you give appropriate credit to the original author(s) and the source, provide a link to the Creative Commons licence, and indicate if changes were made. The images or other third party material in this article are included in the article's Creative Commons licence, unless indicated otherwise in a credit line to the material. If material is not included in the article's Creative Commons licence and your intended use is not permitted by statutory regulation or exceeds the permitted use, you will need to obtain permission directly from the copyright holder. To view a copy of this licence, visit http://creativecommons.org/licenses/by/4.0/. The Creative Commons Public Domain Dedication waiver (http://creativecommons.org/publicdomain/zero/1.0/) applies to the data made available in this article, unless otherwise stated in a credit line to the data. 
to overcome this resistance have focused on the development of inhibitors against dePARylation proteins and more specifically against PARG. Since PARG is responsible for reversing the majority of PARylation, anti-PARG inhibitors (PARGi) have demonstrated the promising potential for killing cancerous cells at an efficacy equitant to PARPi [24, 25]. PARGi like PARPi has shown synthetic lethal phenotype in cells deficient in DDR proteins. Besides, PARG being a monogenic protein unlike the redundant PARP enzyme family, a higher degree of specificity could be achieved with PARGi [26]. Here, we review our current understanding of the dePARylation proteins and focus on the recent advancement of exploiting dePARylation proteins in anti-tumor therapies.

\section{PARylation in DNA damage repair}

PARylation is a transient and reversible protein posttranslational modification that modulates the structural and functional properties of the acceptor proteins during a wide variety of biological processes including DDR, cell stress, transcription, immune response, aging and cell death [3, 4, 27]. However, the well-characterized function of PARylation is its role in the regulation of DNA repair signaling. PARylation is catalyzed by a large family of proteins (17 members in total, from PARP1-PARP4, PARP5a-PARP5b and PARP6-PARP16) known as poly(ADP-ribose) polymerases (PARPs). All PARPs share a huge degree of homology with the founding PARP family member PARP1. PARP1 and PARP2 are dominant PARP family enzymes in the cells and act as the primary sensors of DNA damage [2,3]. PARP1 is the most abundant PARP protein in a cell (1-2 million molecules/cell) accounting for $90 \%$ of cellular PARylation, while PARP2 accounts for the remaining $10 \%[5,28,29]$. Apart from PARP1 and PARP2, additional PARP proteins contribute a minor fraction of PARylation or MARylation, PARP9 and PARP13 lack enzymatic activity [30]. Upon DNA damage, PARP1 physically attaches to the damaged DNA through its $\mathrm{N}$-terminal zinc-finger domains and the interaction activates the $\mathrm{C}$-terminal catalytic domain $[31,32]$. The activated PARP1 then hydrolyzes $\mathrm{NAD}^{+}$, resulting in the polymerization of ADPR units on PARP1 itself as well as a huge number of proteins involved in DDR [33]. PARP1 itself is heavily autoPARylated during PARP1 activation $[34,35]$. During polymerization, $\mathrm{NAD}^{+}$ is hydrolyzed into ADPR and nicotinamide is generated as a side product. The first ADPR is covalently attached to the acceptor proteins usually through an ester linkage [36]. PARylation could involve either the attachment of a single or multiple ADPR moieties. Repeated units of ADPR are polymerized into long PAR chains $(O$-glycosidic bonds) which could attain linear and/or branched conformation. A single PAR chain can polymerize up to 200 residues in each polymer and the branches are incorporated after every 20 to 50 residues [37]. The vast majority of ADPR attachment primarily involves glutamate, aspartate, serine [38], arginine and lysine [39] residues in acceptor proteins. The attachment thus involves an $\mathrm{O}$-glycosidic bond for glutamic acid, aspartic acid and serine, while an $\mathrm{N}$-glycosidic bond is formed on arginine and lysine [40, 41].

PAR chains due to the negatively charged phosphates of ADPR bring a lot of anionic charges to the damaged chromatin and the negative charges alter the chemical and biological properties of the acceptor proteins. The acceptor protein of PARylation includes histones (H1, $\mathrm{H} 2 \mathrm{~A}$, and H2B) [41, 42], DNA protein kinases [43, 44], p53 [45], Ku complex [3, 46], DNA glycosylase 8-oxoguanine glycosylase 1 (OGG1) [47], PCNA [48], RUNX [49], etc. Since DNA is negatively charged; charge repulsion between PAR and DNA modulates the chromatin structure at the damaged loci. Besides, proteins containing PAR binding motifs/domains are recruited to the DNA damage site by the PAR signal itself. These downstream proteins include XRCC1 [50], DNA ligase III [51], CHFR [52] and once recruited, these proteins promote protective DNA damage repair. Thus DNA-damage associated PAR signals act as a docking signal and a scaffold on which a huge number of DDR proteins are assembled which favors efficient and optimal DDR $[3,7]$.

PARP1 enzymatic activities are required for all forms of DNA damage including mismatch repair, base excision repair, SSB repair and DSB repair [28]. PARP2 enzymatic activities are however limited to BER and restarting blocked replication forks [53]. However, our recent studies indicate that PARP2 plays an important role in branched PAR chain synthesis. The frequency of branching was decreased by more than half in PARP2 knockout mice which was rescued by wild type PARP2. We observed that PARP2 mediated branching was initiated by the PAR chain interaction with $\mathrm{N}$-terminus of PARP2 and the branching PAR was important for the recruitment of histone removal proteins (e.g. APLF) during DNA damage repair [5].

Although PARP1 and PARP2 are crucially required for maintaining genomic stability, mice lacking either protein are viable, although these mice are hypertensive to DNA damaging agents $[3,54]$. This discrepancy is attributed to the high redundancy of these two PARP proteins during embryonic development. Thus, the lack of one PARP protein can be complemented by the other. However, synthetic lethality is achieved when both PARP enzymes (e.g. PARP1 an PARP2 [55]) and proteins involved in DDR are inhibited [56]. This property is clinically exploited and used by anti-PARylation inhibitors in cancer therapy and will be discussed in detail later in this review. 


\section{The role of dePARylation in DNA damage repair}

PARylation and MARylation, like other biological modifications, are precisely regulated. The de-polymerization terminates the ADP-ribosylation-associated signaling cascade and steady-state is achieved. PARylation recruits DDR proteins near the damaged loci and dePARylation facilitates their deposition onto the damaged site. Failure to remove the PAR signal will result in the trapping of DDR proteins at the vicinity of the damaged DNA and causing cells hypersensitive to DNA damage [24, 57]. The rapid hydrolysis of PAR happens almost immediately after PAR synthesis is achieved. These hydrolases include PARG, TARG1, MacroD1, MacroD2, ARH1 and ARH3 (Fig. 1). These proteins contain a highly conserved macro domain fold hydrolyzes the glycosidic bond among ADPR units or between ADPR and protein residues [13-15]. PARG is the dominant enzyme involved in PAR chain removal and this activity is only weakly observed in TARG1 and ARH3. TARG1, ARH1, ARH3, MacroD1 and MacroD2 are involved in the removal of proximal ADPR/MAR. Recent studies have implicated two other pyrophosphatases Nudix Hydrolase 16 (NUDT16) and Ectophosphodiesterase/nucleotide phosphohydrolase
(ENPP) that may digest the phosphor-diester bond in ADPR during PAR metabolism.

\section{PARG}

The majority $(\sim 90 \%)$ of the cellular PAR chains are digested by the catabolic enzyme PARG $[58,59]$. Alternative splicing of a single PARG gene product generates five isoforms that have variable size, cellular distribution and activity $[60,61]$. PARG is recruited to the PAR locations by PCNA which is facilitated by acetylation of lysine (K409) on PARG [27, 62]. PARG acts as both endo and exo-glycohydrolase [63], and is well suited to hydrolyze the $O$-glycosidic bonds between the ADPR units of PAR; however, as mentioned earlier, the terminal ADPR is linked to an acceptor protein via an ester bond and consequently resists PARG mediated hydrolysis [12, 64]. Nevertheless, PARG is critically important for cellular function and loss of PARG causes embryonic lethality in mice [65]. Thus, unlike PARP1/PARP2 deletion which can be tolerated by cells to a certain extent, PARG-associated function is required for cell viability. Our recent studies confirmed the role of PARG in DNA damage repair. We observed that shRNA mediated knockdown of PARG

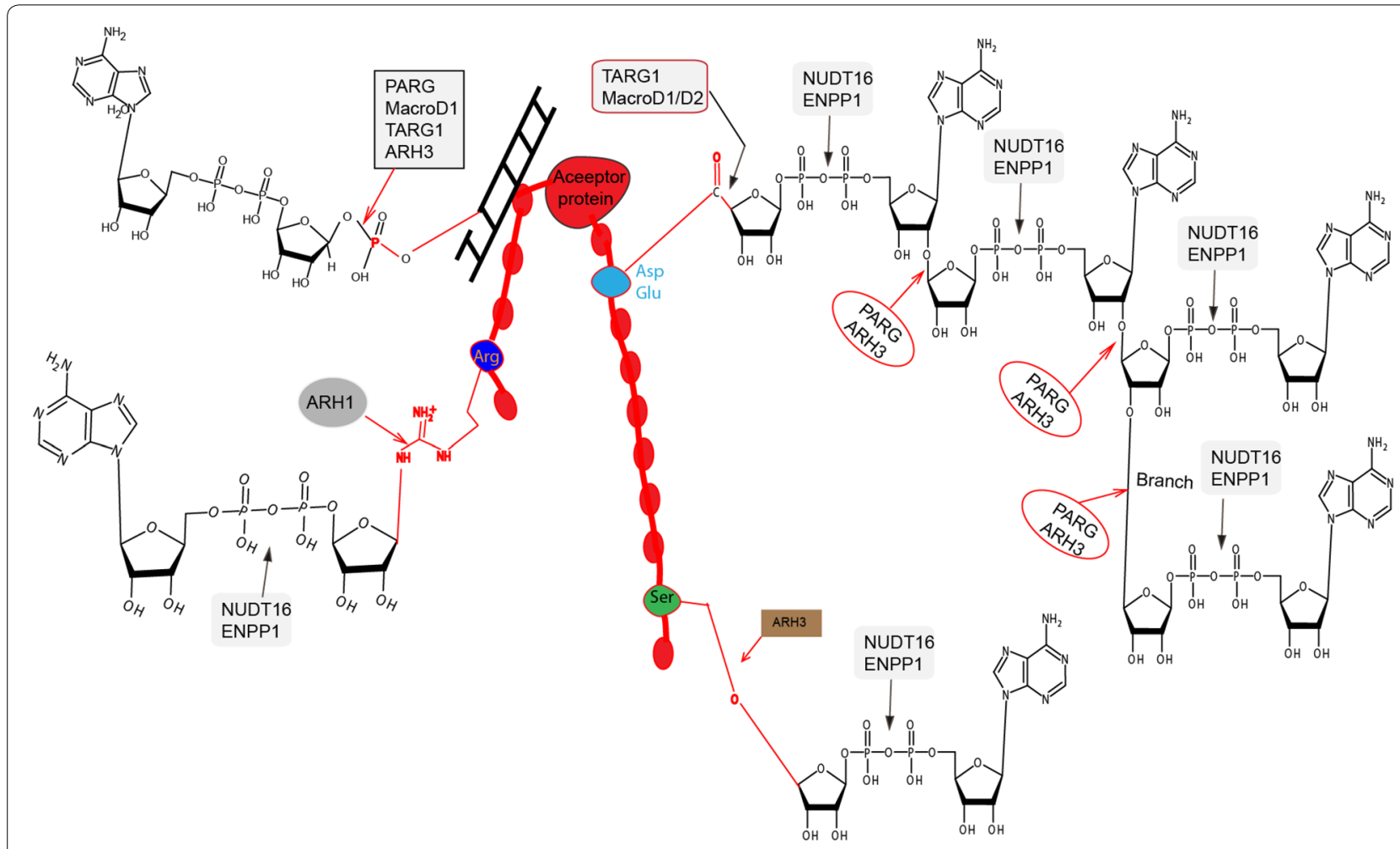

Fig. 1 Schematic diagram showing the proteins involved in dePARylation. The acceptor protein is shown as beads on a string. The dominant amino acids involved in PARylation (i.e. aspartic acid and glutamic acid are shown light blue, arginine is shown in dark blue, serine is shown in light green). A dsDNA helix is and MAR moiety attached to it is shown in black. The bonds hydrolyzed by different dePARylation proteins are shown. A linear and branched PAR chain is shown attached to aspartic acid and glutamic acid 
was associated with defective DNA single-stranded and double-stranded break repair by trapping DDR factors on PAR chains at the damaged loci [24]. Thus, precisely regulated PARP1 and PARG activities promote optimal response to cellular stress conditions.

\section{TARG1}

TARG1 is an 18 7kd Macro domain-containing protein that predominantly hydrolyzes mono-ADPR from aspartate and glutamate. TARG1 is primarily recruited to a damaged site by PAR signals wherein it cleaves the last ADPR moiety from the side chains of aspartate and glutamate residue following PARG-mediated dePARylation $[66,67]$. Thus, one major function of TARG1 is to complete the PAR removal process once PARG direst the rest of PAR chains. In addition, TARG1 has weak PAR removal function probably due to the removal of the whole PAR chain directly from aspartate and glutamate [66].

\section{ARH1 and ARH3}

ARH family enzymes resemble dinitrogenase reductaseactivating Glycohydrolase (DraG) family enzymes that mediate nitrogen fixation in bacteria [68]. ARH1-3 proteins are identical in size $(39 \mathrm{kDa})$ and share similar primary sequences. ARH1 and ARH3 hydrolases primarily act on MAR moieties on the acceptor proteins. ARH1 is mainly involved in the hydrolysis of the $N$-glycosidic bond formed between arginine and ADPR [69]. ARH3 has the strongest hydrolytic activity within the ARH family, which like PARG can hydrolyze PAR chains of the acceptor proteins. However, unlike PARG, which lacks terminal hydrolase activity, ARH3 can remove MAR moieties as well. Recent studies have implicated ARH3 in the hydrolysis of the glycosidic bond between serine and ADPR [70]. Serine-ADP-ribosylation is emerging as an abundant form of protein ADP-ribosylation on DNA damage response proteins primarily catalyzed by PARP1 and PARP2 [71, 72]. ARH2 binds to ADPR but has no reported activity on either MARylated or PARylated proteins. Thus ARH1 and ARH3 proteins, along with TARG1 are required for a complete reversal of PARylation postPARG mediated digestion.

\section{MacroD1 and MacroD2}

These proteins share similar Macro domain fold (also found in PARG and TARG1) probably emerging from gene duplication during evolution. MacroD1 and MacroD2 like TARG1 are required to remove the proximal ADPR from the Asp and Glu residues of the acceptor proteins. They can also function as dominant MAR hydrolases in the cells $[13,15]$. Recent studies suggest that MacroD1 is also involved in the deMARylation of dsDNA [73].

\section{NUDT16 and ENPP}

NUDT16 is a member of Nudix superfamily hydrolases found across all living organisms encompassing archaea, bacteria and eukaryotes. These family enzymes are primarily involved in the digestion of pyrophosphate containing substrates like dNTP, nucleoside diand triphosphates, etc. Interestingly, both PAR chains and MAR can act as a substrate for NUDT16 [74]. However, due to the phosphatase nature of NUDT16 catalysis, ribose- $5^{\prime}$-phosphate is retained on the acceptor proteins and phosphoribosyl-AMP is released. Thus complete reversal of PARylation/MARylation cannot be achieved by NUDT16 and the proteins required for removing the NUDT16 signature sequence are not known [9, 75]. ENPP is a recently characterized PAR/ MAR phosphodiesterase and like NUDT16 catalysis, ENPP mediated catalysis is characterized by retention of ribose-5'-phosphate on acceptor proteins at the PAR/ MAR attachment site [76].

\section{PARP inhibitors in cancer treatment}

PARylation is responsible for both initial sensing of DNA damage and the recruitment of DNA damage response proteins to the damaged site. Consequently, disruption of this crucial cellular signal is associated with the accumulation of DNA lesions, which causes cell death. However, as discussed earlier, despite its critical role in maintaining genomic integrity, PARP1/PARP2 knockout does not induce lethality. This is attributed to the redundant function of PARP proteins as well as multiple DNA damage repair pathways. However, cells deficient in the alternative DNA repair pathways/proteins undergo apoptosis upon PARP1/PARP2 inhibition. This feature is known as synthetic lethality, a phenomenon in which cells with one defect i.e. either a mutation in DDR or PARP inhibition can survive, but a combination of the two together causes cell death [77-79].

PARPi mediated synthetic lethality has been exploited in clinical cancer treatment. PARPi including olaparib, rucaparib, niraparib, talazoparib act by both inhibiting PAR formation as well as by blocking the PARP1 release from the damaged DNA. These inhibitors are PARP1/2 competitive inhibitors and compete with the cellular $\mathrm{NAD}^{+}$for binding to PARP1 [80]. Moreover, the inhibitors trap PARP1/2 on the sites of damaged DNA forming PARP-DNA complexes and the stalled replication forks cause cell death [57]. Olaparib was the first PARPi to receive FDA approval for the treatment of advanced ovarian cancer with BRCA mutations in 2014. Over the past few years, olaparib has been extended and FDA 
approved in treatments of other cancers including triplenegative breast cancer and pancreatic cancer with BRCA mutations.

PARP inhibitors have developed into promising and potent therapeutic strategies against a wide variety of cancers. However, unfortunately, like with other cellular therapies, PARPi resistance has emerged in the clinic [21, 81]. Apart from increased drug efflux, these resistance mechanisms involve reverent mutations in BRCA1/2 genes. These reverse mutations produce normal protein and the cells switch to normal HR in presence of PARPi. Besides, PARP1 protein itself may be lost from the cells under prolonged PARPi treatment. Moreover, cells may partially restore PARylation by inactivating PAR digesting enzyme PARG [82]. An additional mechanism of resistance involves the inactivation of critical DDR proteins like 53BP1, SLFN11 [83], REV7, EZH2, BRD7 [84], EMI1 [85], etc. Recent studies have indicated that PARPi ovarian cancer cells can attain resistance to PARPi due to enhanced microhomology-mediated end joining attributed to increased expression of ALDH1A1 [86] and due to decreased $\mathrm{m}^{6} \mathrm{~A}$ levels on FZD10 mRNA which in turn activates Wnt signaling pathway [87].

\section{Developing PARG inhibitors}

DePARylation is equally important for proper cell function as PARylation. Complete coordination of the two processes is essential for proper DNA damage response. We and others have shown that dePARylation is not merely an antagonistic process of PARylation in the context of DNA damage repair. Instead, dePARylation is an immediately downstream step of PARylation. The function of PARylation is to mediate the recruitment of DDR factors to the proximity of DNA lesions, whereas dePARylation releases these DDR factors from PAR chains, so that these factors can be loaded at exact DNA lesions for repair. Suppression of dePARylation traps DDR factors onto the PAR chains, thus impairs SSB and DSB repair [24]. Moreover, therapeutic inhibition of PARP enzymes is compounded due to the presence of multiple PARP isoforms. Since, mice lacking PARP1 or PARP2 are viable, double knockouts of PARP1 and PARP2 leads to the death of the mice [55]. This phenotype of the double knockout mice suggests that the two proteins play a redundant role and in the absence of one PARP (e.g. PARP1) the function can be compensated by other PARP protein (e.g. PARP2). Accordingly, during therapeutic inhibition of one PARP enzyme, uninhibited enzymes may compensate for the lost PARP protein.

Targeting of dePARylation may circumvent some of the problems associated with PARPi resistance. PARG unlike multiple PARP proteins is monogenic and does not share its dePARylation function. Consequently, higher potency and specificity could be achieved with PARG inhibition [1, 3] (Table 1 and Fig. 2). In addition, PARPi resistance involves loss of PARP1 itself from the resistant cells. However, a similar PARG loss is unlikely due to the cell lethal phenotype of the PARG deficient cells.

The first generations of PARG inhibitors were DNA intercalating polyaromatic molecules such as proflavine, ethidium bromide and ethacridine. These inhibitors directly bind the PAR chain thereby blocking PARG mediated hydrolysis of the polymer [88]. The potency of intercalators was enhanced with the introduction of two more PARG inhibitors GPI 16,552 and 18,214 and the inhibitors were presented as potent PARG inhibitors against ant-inflammatory protection [89]. The next class of PARG inhibitors was tannins (e.g. Nobotanin K) [90] and ADP-ribose analogs (e.g. Adenosine diphosphate (hydroxymethyl)pyrrolidinediol (ADP-HPD) [91]. These

Table 1 PARG inhibitors and their drawbacks

\begin{tabular}{|c|c|c|c|c|}
\hline Inhibitor & Activity & Limitations and $I C_{50}$ & Cancer type/model & References \\
\hline $\begin{array}{l}\text { Intercalating molecules, e.g. } \\
\text { proflavine, ethidium bromide, } \\
\text { ethacridine }\end{array}$ & $\begin{array}{l}\text { Bind PAR and resist PARG mediated } \\
\text { hydrolysis }\end{array}$ & $\begin{array}{l}\text { Not effective in-vitro and cell imper- } \\
\text { meable, } \mathrm{IC}_{50}>7 \mu \mathrm{M}\end{array}$ & Ex-vivo & {$[11,88]$} \\
\hline GPI16552 and GPI 18214 & Same as above & Not effective in-vitro, $I C_{50}>1.7 \mu \mathrm{M}$ & Colon tissue mice & {$[11,89]$} \\
\hline Tannins e.g. Nobotanin K & $\begin{array}{l}\text { ADPr analogs i.e. bind PAR and resist } \\
\text { PARG }\end{array}$ & Low cell permeability, $I C_{50}>0.3 \mu \mathrm{M}$ & Cell line & {$[11,90]$} \\
\hline Salicylanilides & Bind PARG and inhibit dePARylation & $\begin{array}{l}\text { Not effective in-vivo and non-specifi- } \\
\text { cally (inhibit PARP1), } I C_{50}>12 \mu \mathrm{M}\end{array}$ & Cell line & {$[92]$} \\
\hline RBPIs & Block PARG mediated PAR hydrolysis & $\begin{array}{l}\text { Low specificity and less potency, } \\
\qquad \mathrm{IC}_{50}>2.9 \mu \mathrm{M}\end{array}$ & Cell line & {$[93]$} \\
\hline PDD00017273 & $\begin{array}{l}\text { Replication fork stalling and low DNA } \\
\text { double stranded break repair }\end{array}$ & Low metabolic activity, $I_{50}>25$ nM, & Cell line & {$[25,96]$} \\
\hline $\mathrm{COH} 34$ & $\begin{array}{l}\text { Binds PARG catalytic site and traps } \\
\text { DDR proteins }\end{array}$ & $\mathrm{IC}_{50}=0.37$ & Mice & {$[24]$} \\
\hline
\end{tabular}




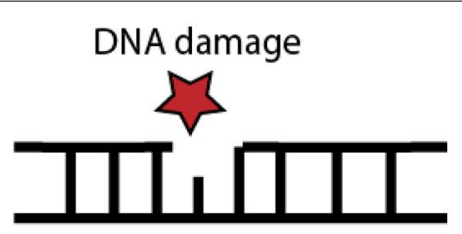

PARP1/2 recruitment followed by autoPARylation and binding of DDR proteins
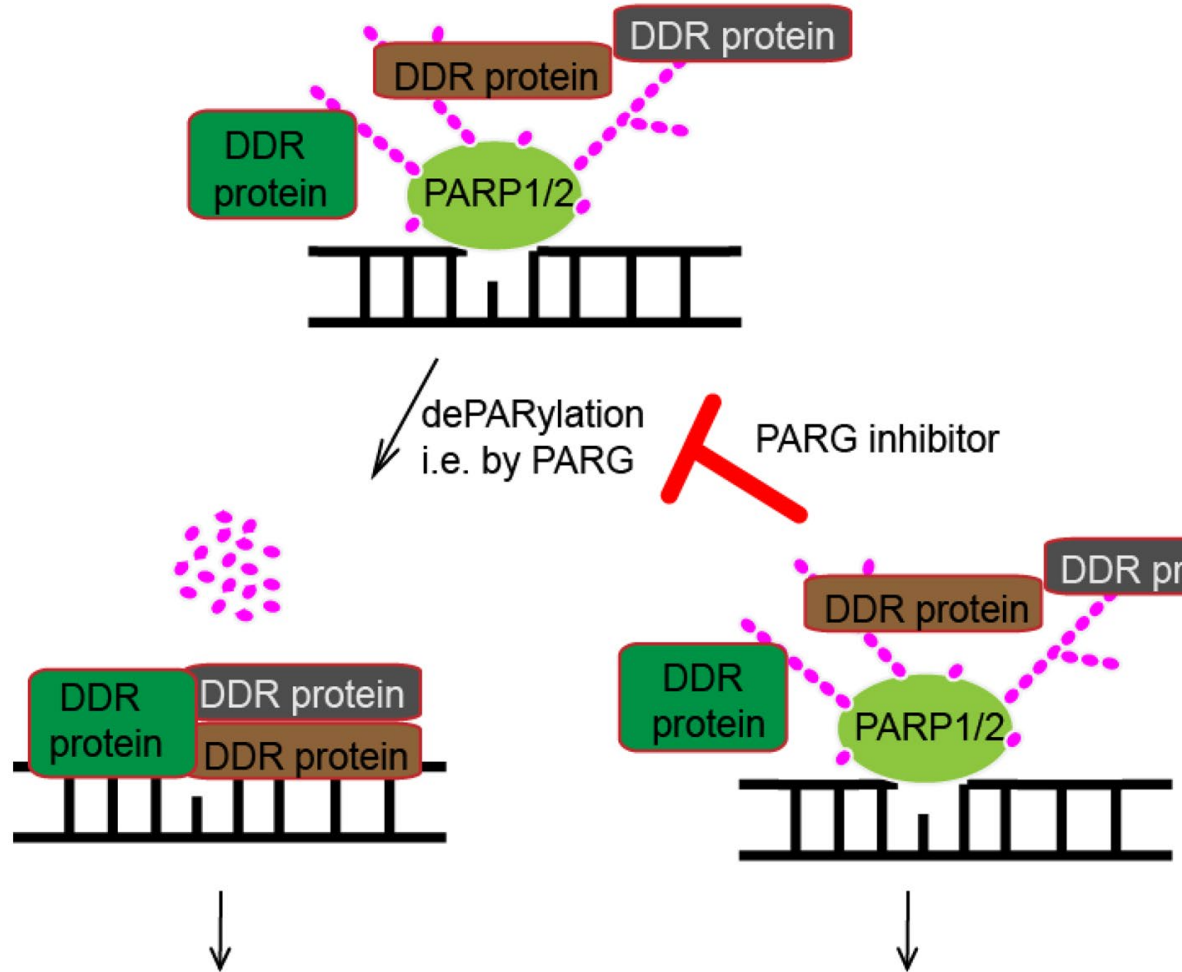

Recruitment of DDR proteins on the damged loci

Trapping of DDR proteins on the PAR<smiles>C[AlH]</smiles>

DNA repair and cell survival

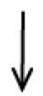

Cell death

Fig. 2 Schematic diagram showing the effect of dePARylation inhibitors on DDR. A damaged (star) DNA is shown on the top. PARP proteins (PARP1/2) are recruited to the site where they undergo PARylation and MARylation. The PAR/MAR moieties recruit DDR proteins to the vicinity of the damaged site. DePARylation (i.e. by PARG) digests the PAR chains, relives the PARP proteins and loads the DDR on the damaged site. DePARylation inhibitors trap the DDR on the PAR chains thereby preventing DDR and leading to cell death

inhibitors were effective in-vitro but lacked cell permeability which would prevent their use on cells.

The first cell-permeable PARGi were identified via a high throughput screening known as salicylanilides which were originally used in fungicide treatments. These inhibitors had an added advantage of inhibiting both PARG as well as PARP1 [92]. Yet another class of synthetic inhibitors based on Rhodamine was developed known as rhodanine-based PARG inhibitors (RBPIs). These inhibitors (e.g. RBPI-1) were specific to PARG with no effect on ARH3 or PARP1 [93]. The inhibitors were more potent than salicylanilides exhibiting high specificity and cell permeability [92]. However, therapeutic testing of these inhibitors was associated with low specificity, less potency and thus with low druglikeness [25]. 


\section{Application of PARG inhibitors in cancer treatment}

As mentioned earlier, PARG inhibition can circumvent the majority of problems associated with PARP1 inhibition. Additionally, recent studies have indicated that increased PARG expression is associated with higher incidences of breast cancers as well as cellular transformation and invasion in vivo [94]. Moreover, PARG suppression has been implicated to prevent lung cancer in PARG $^{+/-}$mice treated with benzo(a)pyrene by stabilizing the expression of Wnt ligand [95]. These observations make PARG a perfect target for cancer chemotherapy. To address the problems associated with previously known PARG inhibitors, D.I. James and colleagues performed high-throughput screening and developed a cell-permeable PARG inhibitor PDD00017273. PDD00017273 caused dose-dependent inhibition of PARG and significant PARG inhibition could be achieved at low concentrations $(0.3 \mu \mathrm{M})$. When breast cancer MCF7 cells were treated with PDD00017273, the cells showed increased DNA damage consistent with increased $\gamma \mathrm{H} 2 \mathrm{AX}$ formation and reduced cell survival [25]. Inhibitor treatment induced the replication fork stalling and favored DNA repair via HR. Consequently, PDD00017273 like olaparib exhibited synthetic lethal phenotype in cells deficient in HR proteins BRCA1/2, PALB2, BARD1 etc. [96, 97]. Recently, PDD00017273 was used successfully in vitro against pancreatic ductal adenocarcinoma (PDAC) cells as a monotherapy and combination therapy [98]. However, due to poor metabolic activity, this PARG inhibitor cannot be used for cancer treatment in vivo.

We recently discovered a novel PARG inhibitor deciphering the highest potency, cell permeability and tumor cell lethality [24]. The compound known as $\mathrm{COH} 34$ inhibits PARG at nanomolar concentrations and induced tumor cell lethality both in vitro and in vivo. $\mathrm{COH} 34$ was highly potent with an $\mathrm{IC}_{50}$ value of $0.37 \mathrm{nM}$. COH34 mediated inhibition is highly specific to PARG and there is no-cross inhibition of other dePARylation enzymes such as TARG1 and ARH3. COH34 and PARG had a binding ratio of $1: 1$ wherein $\mathrm{COH} 34$ binds snugly into the catalytic pocket of PARG and thus competes with its normal substrate i.e. PAR. The extended PARylation in turn trapped DDR proteins (like XRCC1, APLF and CHFR) at the damaged site and thereby blocking normal DDR. COH34 exhibited synthetic lethality in cells deficient in BRCA1/BRCA1 and even those cells resistant to olaparib with its inhibitory potential exceeding olaparib. Finally, we characterized and validated the efficacy of $\mathrm{COH} 34$ against tumors with DDR defects in vivo. Moreover, $\mathrm{COH} 34$ was stable in vivo and non-toxic to mice at $20 \mathrm{mg} / \mathrm{kg}$ concentration [24]. Collectively, COH34 is a very promising lead compound for the development of dePARylation inhibitors for cancer treatment.

\section{Conclusion}

Therapeutic targeting of PARylation and dePARylation represents an ideal target in cancer chemotherapy. Encouraged by the successful FDA approval and clinical use of olaparib, several anti-PARylation drugs are at different stages of clinical trials. Moreover, the utility of these drugs is expanding beyond breast cancer to other cancer including those in the ovary, prostate, pancreas, etc. Additional synthetic lethalities have been reported. However, the emergence of resistance against PARPi necessities the development of alternative therapeutic strategies. In this direction, a recent class of PARP1 inhibitors was developed which can degrade PARP1 (e.g. iRucaparib-AP6) with high potency and specificity [99]. However, additional studies are needed to demonstrate the therapeutic potential of these inhibitors.

DePARylation inhibitors like $\mathrm{COH} 34$ represent a novel class of inhibitors alternative to PARP inhibition, and may overcome chemo-resistance of PARPi. The inhibition of PARG by COH34 along with other inhibitors would be particularly effective due to the monogenic character of PARG. The possibility of resistance arising due to the redundant nature of PARP proteins could be particularly avoided with these inhibitors. Further development of dePARylation inhibitor into clinical cancer treatment may generate a huge impact on cancer patients.

\section{Acknowledgements \\ We thank our colleagues at City of Hope for supportive discussions \\ Authors' contributions \\ MAK, LLY, XY wrote the paper. All authors read and approved the final manuscript. \\ Funding \\ This work was supported by Grants from the National Institutes of Health (CA130899 and CA240392) to X.Y. X.Y. is a recipient of Research Scholar Award from Tower Cancer Research Foundation, Taub Foundation and Pancreatic Cancer Action Network.}

Availability of data and materials

Not applicable.

Ethics approval and consent to participate Not applicable.

Consent for publication

All authors agree with the publication of the final version of the manuscript.

Competing interests

The authors declare that they have no competing interests.

\section{Author details}

1 Department of Cancer Genetics \& Epigenetics, Beckman Research Institute, City of Hope, Duarte, CA 91010, USA. ${ }^{2}$ Westridge School, 324 Madeline Dr., Pasadena, CA 91105, USA.

Received: 5 December 2019 Accepted: 9 January 2020

Published online: 29 January 2020 


\section{References}

1. Kassab MA, Yu X. The role of dePARylation in DNA damage repair and cancer suppression. DNA Repair. 2019;76:20-9.

2. Gibson BA, Kraus WL. New insights into the molecular and cellular functions of poly(ADP-ribose) and PARPs. Nat Rev Mol Cell Biol. 2012;13:411-24.

3. Liu C, Vyas A, Kassab MA, Singh AK, Yu X. The role of poly ADP-ribosylation in the first wave of DNA damage response. Nucleic Acids Res. 2017:45:8129-41.

4. Gupte R, Liu Z, Kraus WL. PARPs and ADP-ribosylation: recent advances linking molecular functions to biological outcomes. Genes Dev. 2017:31:101-26.

5. Chen Q, Kassab MA, Dantzer F, Yu X. PARP2 mediates branched poly ADPribosylation in response to DNA damage. Nat Commun. 2018;9:3233.

6. Schreiber V, Dantzer F, Ame J-C, de Murcia G. Poly(ADP-ribose): novel functions for an old molecule. Nat Rev Mol Cell Biol. 2006;7:517-28.

7. Wei H, Yu X. Functions of PARylation in DNA damage repair pathways. Genom Proteom Bioinform. 2016;14:131-9.

8. Palazzo L, Mikoč A, Ahel I. ADP-ribosylation: new facets of an ancient modification. FEBS J. 2017:284:2932-46.

9. Daniels CM, Thirawatananond P, Ong S-E, Gabelli SB, Leung AKL. Nudix hydrolases degrade protein-conjugated ADP-ribose. Sci Rep. 2015;5:18271.

10. Bonicalzi M-E, Haince J-F, Droit A, Poirier GG. Regulation of poly(ADPribose) metabolism by poly(ADP-ribose) glycohydrolase: where and when? Cell Mol Life Sci. 2005;62:739-50.

11. Feng $X, K o h$ DW. Roles of poly(ADP-ribose) glycohydrolase in DNA damage and apoptosis. Int Rev Cell Mol Biol. 2013;304:227-81.

12. Slade $D$, et al. The structure and catalytic mechanism of a poly(ADPribose) glycohydrolase. Nature. 2011;477:616-20.

13. Jankevicius $\mathrm{G}$, et al. A family of macrodomain proteins reverses cellular mono-ADP-ribosylation. Nat Struct Mol Biol. 2013;20:508-14.

14. Rosenthal F, et al. Macrodomain-containing proteins are new mono-ADPribosylhydrolases. Nat Struct Mol Biol. 2013;20:502-7.

15. Rack JGM, Perina D, Ahel I. Macrodomains: structure, function, evolution, and catalytic activities. Annu Rev Biochem. 2016;85:431-54.

16. Lord CJ, Ashworth A. PARP inhibitors: synthetic lethality in the clinic. Science. 2017:355:1152-8.

17. Ashworth A, Lord CJ. Synthetic lethal therapies for cancer: what's next after PARP inhibitors? Nat Rev Clin Oncol. 2018;15:564-76.

18. Murai J, et al. Trapping of PARP1 and PARP2 by clinical PARP inhibitors. Cancer Res. 2012;72:5588-99.

19. Murai J, et al. Stereospecific PARP trapping by BMN 673 and comparison with olaparib and rucaparib. Mol Cancer Ther. 2014;13:433-43.

20. Fong PC, et al. Inhibition of poly(ADP-ribose) polymerase in tumors from BRCA mutation carriers. N Engl J Med. 2009;361:123-34.

21. Noordermeer SM, van Attikum H. PARP inhibitor resistance: a tug-of-war in BRCA-mutated cells. Trends Cell Biol. 2019;29:820-34.

22. Montoni A, Robu M, Pouliot É, Shah GM. Resistance to PARP-inhibitors in cancer therapy. Front Pharmacol. 2013;4:18.

23. Gogola E, Rottenberg S, Jonkers J. Resistance to PARP inhibitors: lessons from preclinical models of BRCA-associated cancer. Annu Rev Cancer Biol. 2019:3:235-54

24. Chen S-H, Yu X. Targeting dePARylation selectively suppresses DNA repair-defective and PARP inhibitor-resistant malignancies. Sci Adv. 2019;5:eaav4340

25. James Dl, et al. First-in-class chemical probes against poly(ADP-ribose) glycohydrolase (PARG) inhibit DNA repair with differential pharmacology to olaparib. ACS Chem Biol. 2016;11:3179-90.

26. Meyer RG, Meyer-Ficca ML, Jacobson EL, Jacobson MK. Human poly(ADPribose) glycohydrolase (PARG) gene and the common promoter sequence it shares with inner mitochondrial membrane translocase 23 (TIM23). Gene. 2003;314:181-90.

27. Kaufmann T, et al. A novel non-canonical PIP-box mediates PARG interaction with PCNA. Nucleic Acids Res. 2017:45:9741-59.

28. Amé J-C, Spenlehauer C, de Murcia G. The PARP superfamily. BioEssays News Rev Mol Cell Dev Biol. 2004;26:882-93.

29. Woodhouse BC, Dianov GL. Poly ADP-ribose polymerase-1: an international molecule of mystery. DNA Repair. 2008;7:1077-86.
30. Hottiger MO, Hassa PO, Lüscher B, Schüler H, Koch-Nolte F. Toward a unified nomenclature for mammalian ADP-ribosyltransferases. Trends Biochem Sci. 2010:35:208-19.

31. Langelier M-F, Planck JL, Roy S, Pascal JM. Structural basis for DNA damage-dependent poly(ADP-ribosyl)ation by human PARP-1. Science. 2012;336:728-32.

32. Ali AAE, et al. The zinc-finger domains of PARP1 cooperate to recognize DNA strand breaks. Nat Struct Mol Biol. 2012;19:685-92.

33. Alemasova EE, Lavrik OI. Poly(ADP-ribosyl)ation by PARP1: reaction mechanism and regulatory proteins. Nucleic Acids Res. 2019;47:3811-27.

34. Kim MY, Mauro S, Gévry N, Lis JT, Kraus WL. NAD+-dependent modulation of chromatin structure and transcription by nucleosome binding properties of PARP-1. Cell. 2004;119:803-14.

35. Wacker DA, et al. The DNA binding and catalytic domains of poly(ADPribose) polymerase 1 cooperate in the regulation of chromatin structure and transcription. Mol Cell Biol. 2007:27:7475-85.

36. de Murcia G, Ménissier de Murcia J. Poly(ADP-ribose) polymerase: a molecular nick-sensor. Trends Biochem Sci. 1994;19:172-6.

37. Alvarez-Gonzalez R, Jacobson MK. Characterization of polymers of adenosine diphosphate ribose generated in vitro and in vivo. Biochemistry. 1987;26:3218-24

38. Larsen SC, Hendriks IA, Lyon D, Jensen LJ, Nielsen ML. Systems-wide analysis of serine ADP-ribosylation reveals widespread occurrence and site-specific overlap with phosphorylation. Cell Rep. 2018;24:2493-2505. e4.

39. Messner S, et al. PARP1 ADP-ribosylates lysine residues of the core histone tails. Nucleic Acids Res. 2010;38:6350-62.

40. Honjo T, Kinoshita K, Muramatsu M. Molecular mechanism of class switch recombination: linkage with somatic hypermutation. Annu Rev Immunol. 2002;20:165-96.

41. Zhang Y, Wang J, Ding M, Yu Y. Site-specific characterization of the Aspand Glu-ADP-ribosylated proteome. Nat Methods. 2013;10:981-4.

42. Krishnakumar $\mathrm{R}$, et al. Reciprocal binding of PARP-1 and histone $\mathrm{H} 1$ at promoters specifies transcriptional outcomes. Science. 2008;319:819-21.

43. Falck J, Coates J, Jackson SP. Conserved modes of recruitment of ATM, ATR and DNA-PKcs to sites of DNA damage. Nature. 2005:434:605-11.

44. Shiloh Y, Ziv Y. The ATM protein kinase: regulating the cellular response to genotoxic stress, and more. Nat Rev Mol Cell Biol. 2013;14:197-21010.

45. Matsuoka S, et al. ATM and ATR substrate analysis reveals extensive protein networks responsive to DNA damage. Science. 2007;316:1160-6.

46. Couto CA-M, et al. PARP regulates nonhomologous end joining through retention of Ku at double-strand breaks. J Cell Biol. 2011:194:367-75.

47. Noren Hooten N, Kompaniez K, Barnes J, Lohani A, Evans MK. Poly(ADPribose) polymerase 1 (PARP-1) binds to 8-oxoguanine-DNA glycosylase (OGG1). J Biol Chem. 2011;286:44679-90.

48. Simbulan-Rosenthal CM, et al. The expression of Poly(ADP-ribose) polymerase during differentiation-linked DNA replication reveals that it is a component of the multiprotein DNA replication complex. Biochemistry. 1996:35:11622-33.

49. Tay LS, et al. RUNX Poly(ADP-Ribosyl)ation and BLM interaction facilitate the fanconi anemia pathway of DNA repair. Cell Rep. 2018;24:1747-55.

50. Masson $\mathrm{M}$, et al. XRCC1 is specifically associated with poly(ADP-ribose) polymerase and negatively regulates its activity following DNA damage. Mol Cell Biol. 1998;18:3563-71.

51. Okano S, Lan L, Tomkinson AE, Yasui A. Translocation of XRCC1 and DNA ligase Illalpha from centrosomes to chromosomes in response to DNA damage in mitotic human cells. Nucleic Acids Res. 2005;33:422-9.

52. Ahel l, et al. Poly(ADP-ribose)-binding zinc finger motifs in DNA repair/ checkpoint proteins. Nature. 2008;451:81-5.

53. Schreiber $V$, et al. Poly(ADP-ribose) polymerase-2 (PARP-2) is required for efficient base excision DNA repair in association with PARP-1 and XRCC1. J Biol Chem. 2002;277:23028-36.

54. Wang ZQ, et al. Mice lacking ADPRT and poly(ADP-ribosyl)ation develop normally but are susceptible to skin disease. Genes Dev. 1995;9:509-20.

55. Ménissier de Murcia J, Ricoul M, Tartier L, Niedergang C, Huber A, Dantzer F, Schreiber V, Amé J-C, Dierich A, LeMeur M, Sabatier L, Chambon P, De Murcia G. Functional interaction between PARP-1 and PARP-2 in chromosome stability and embryonic development in mouse. EMBO J. 2003. https://doi.org/10.1093/emboj/cdg206. 
56. De Vos M, Schreiber V, Dantzer F. The diverse roles and clinical relevance of PARPs in DNA damage repair: current state of the art. Biochem Pharmacol. 2012;84:137-46.

57. Murai J, et al. Differential trapping of PARP1 and PARP2 by clinical PARP inhibitors. Cancer Res. 2012;72:5588-99.

58. Fisher AEO, Hochegger H, Takeda S, Caldecott KW. Poly(ADP-ribose) polymerase 1 accelerates single-strand break repair in concert with poly(ADP-ribose) glycohydrolase. Mol Cell Biol. 2007;27:5597-605.

59. Min W, Wang Z-Q. Poly (ADP-ribose) glycohydrolase (PARG) and its therapeutic potential. Front Biosci. 2009;14:1619-26.

60. Meyer-Ficca ML, Meyer RG, Coyle DL, Jacobson EL, Jacobson MK. Human poly(ADP-ribose) glycohydrolase is expressed in alternative splice variants yielding isoforms that localize to different cell compartments. Exp Cell Res. 2004:297:521-32.

61. Meyer RG, Meyer-Ficca ML, Whatcott CJ, Jacobson EL, Jacobson MK. Two small enzyme isoforms mediate mammalian mitochondrial poly(ADPribose) glycohydrolase (PARG) activity. Exp Cell Res. 2007;313:2920-36.

62. Mortusewicz O, Fouquerel E, Amé J-C, Leonhardt H, Schreiber V. PARG is recruited to DNA damage sites through poly(ADP-ribose)- and PCNAdependent mechanisms. Nucleic Acids Res. 2011;39:5045-56.

63. Hatakeyama K, Nemoto Y, Ueda K, Hayaishi O. Purification and characterization of poly(ADP-ribose) glycohydrolase. Different modes of action on large and small poly(ADP-ribose). J Biol Chem. 1986;261:14902-11.

64. Oka S, Kato J, Moss J. Identification and characterization of a mammalian 39-kDa poly(ADP-ribose) glycohydrolase. J Biol Chem. 2006;281:705-13.

65. Koh DW, et al. Failure to degrade poly(ADP-ribose) causes increased sensitivity to cytotoxicity and early embryonic lethality. Proc Natl Acad Sci USA. 2004;101:17699-704.

66. Sharifi R, et al. Deficiency of terminal ADP-ribose protein glycohydrolase TARG1/C6orf130 in neurodegenerative disease. EMBO J. 2013;32:1225-377.

67. Bütepage M, et al. Nucleolar-nucleoplasmic shuttling of TARG1 and its control by DNA damage-induced poly-ADP-ribosylation and by nucleolar transcription. Sci Rep. 2018;8:6748.

68. Mashimo M, Kato J, Moss J. Structure and function of the ARH family of ADP-ribose-acceptor hydrolases. DNA Repair. 2014;23:88-94.

69. Laing S, Unger M, Koch-Nolte F, Haag F. ADP-ribosylation of arginine. Amino Acids. 2011:41:257-69.

70. Fontana P, et al. Serine ADP-ribosylation reversal by the hydrolase ARH3. eLife. 2017. https://doi.org/10.7554/eLife.28533.

71. Abplanalp J, et al. Proteomic analyses identify ARH3 as a serine monoADP-ribosylhydrolase. Nat Commun. 2017;8:2055.

72. Bonfiglio JJ, Colby T, Matic I. Mass spectrometry for serine ADP-ribosylation? Think o-glycosylation! Nucleic Acids Res. 2017;45:6259-64.

73. Agnew T, et al. MacroD1 is a promiscuous ADP-ribosyl hydrolase localized to mitochondria. Front Microbiol. 2018;9:20.

74. Palazzo L, et al. Processing of protein ADP-ribosylation by Nudix hydrolases. Biochem J. 2015;468:293-301.

75. Williams JC, Chambers JP, Liehr JG. Glutamyl ribose 5-phosphate storage disease. A hereditary defect in the degradation of poly(ADP-ribosylated) proteins. J Biol Chem. 1984;259:1037-42.

76. Palazzo L, et al. ENPP1 processes protein ADP-ribosylation in vitro. FEBS J. 2016;283:3371-88.

77. Bryant HE, et al. Specific killing of BRCA2-deficient tumours with inhibitors of poly(ADP-ribose) polymerase. Nature. 2005:434:913-7.

78. Farmer $\mathrm{H}$, et al. Targeting the DNA repair defect in BRCA mutant cells as a therapeutic strategy. Nature. 2005;434:917-21.

79. Dobzhansky Th. genetics of natural populations Xiii recombination and variability in populations of drosophila pseudoobscura. Genetics. 1946:31:269-90
80. Langelier M-F, Zandarashvili L, Aguiar PM, Black BE, Pascal JM. NAD+ analog reveals PARP-1 substrate-blocking mechanism and allosteric communication from catalytic center to DNA-binding domains. Nat Commun. 2018:9:844.

81. D'Andrea AD. Mechanisms of PARP inhibitor sensitivity and resistance. DNA Repair. 2018;71:172-6.

82. Gogola E, et al. Selective loss of PARG restores PARylation and counteracts PARP inhibitor-mediated synthetic lethality. Cancer Cell. 2018;33:10781093.e12.

83. Murai J, et al. Resistance to PARP inhibitors by SLFN11 inactivation can be overcome by ATR inhibition. Oncotarget. 2016;7:76534-50.

84. Hu K, et al. Poly(ADP-ribosyl)ation of BRD7 by PARP1 confers resistance to DNA-damaging chemotherapeutic agents. EMBO Rep. 2019. https://doi. org/10.15252/embr.201846166.

85. Marzio A, et al. The F-box domain-dependent activity of EMI1 regulates PARPi sensitivity in triple-negative breast cancers. Mol Cell. 2019;73:224237.e6.

86. Liu $\mathrm{L}$, et al. ALDH1A1 contributes to PARP inhibitor resistance via enhancing DNA repair in BRCA2-/- ovarian cancer cells. Mol Cancer Ther. 2019. https://doi.org/10.1158/1535-7163.MCT-19-0242.

87. Fukumoto T, et al. N6-methylation of adenosine of FZD10 mRNA contributes to PARP inhibitor resistance. Cancer Res. 2019;79:2812-20.

88. Tavassoli M, Tavassoli MH, Shall S. Effect of DNA intercalators on poly(ADPribose) glycohydrolase activity. Biochim Biophys Acta. 1985;827:228-34.

89. Cuzzocrea S, et al. Role of poly(ADP-ribose) glycohydrolase in the development of inflammatory bowel disease in mice. Free Radic Biol Med. 2007:42:90-105.

90. Tsai YJ, et al. Mouse mammary tumor virus gene expression is suppressed by oligomeric ellagitannins, novel inhibitors of poly(ADP-ribose) glycohydrolase. J Biol Chem. 1992;267:14436-42.

91. Slama JT, et al. Specific inhibition of poly(ADP-ribose) glycohydrolase by adenosine diphosphate (hydroxymethyl)pyrrolidinediol. J Med Chem. 1995;38:389-93.

92. Steffen JD, Coyle DL, Damodaran K, Beroza P, Jacobson MK. Discovery and structure-activity relationships of modified salicylanilides as cell permeable inhibitors of poly(ADP-ribose) glycohydrolase (PARG). J Med Chem. 2011;54:5403-13.

93. Finch KE, Knezevic CE, Nottbohm AC, Partlow KC, Hergenrother PJ. Selective small molecule inhibition of poly(ADP-ribose) glycohydrolase (PARG). ACS Chem Biol. 2012;7:563-70.

94. Marques M, et al. Oncogenic activity of poly (ADP-ribose) glycohydrolase. Oncogene. 2019:38:2177-91.

95. Dai W, et al. Regulation of Wnt Singaling Pathway by poly(ADP-ribose) glycohydrolase (PARG) silencing suppresses lung cancer in mice induced by benzo(a)pyrene inhalation exposure. Front. Pharmacol. 2019;10:338.

96. Gravells P, Grant E, Smith KM, James DI, Bryant HE. Specific killing of DNA damage-response deficient cells with inhibitors of poly(ADP-ribose) glycohydrolase. DNA Repair. 2017;52:81-91.

97. Fathers C, Drayton RM, Solovieva S, Bryant HE. Inhibition of poly(ADPribose) glycohydrolase (PARG) specifically kills BRCA2-deficient tumor cells. Cell Cycle. 2012;11:990-7.

98. Jain A, et al. Poly (ADP) Ribose glycohydrolase can be effectively targeted in pancreatic cancer. Cancer Res. 2019;79:4491-502.

99. Wang S, et al. Uncoupling of PARP1 trapping and inhibition using selective PARP1 degradation. Nat Chem Biol. 2019;15:1223-311.

\section{Publisher's Note}

Springer Nature remains neutral with regard to jurisdictional claims in published maps and institutional affiliations. 